\title{
Vielfalt und interdisziplinärer Austausch
}

Ein modernes Kongressumfeld und mehr Platz für neue Veranstaltungsformate: Ab 2018 findet der RadiologieKongressRuhr (RKR) in den Westfalenhallen in Dortmund statt. Ob Onkologie oder strukturierte Befundung, Neuroradiologie und Sportverletzungen: Das umfangreiche Programm bietet auch 2018 für alle Gäste ein attraktives Programm, von Studierenden über MTRA bis hin zu Chefärzten.

Die digitale Revolution in der Radiologie Hoffnungsträger oder Hype, Angstmacher oder Lebensretter? In der Eröffnungssession wird ein Blick in die nicht allzu ferne Zukunft geworfen. Viele der RKR-Veranstaltungen laden ein, mitzudiskutieren, beispielsweise das Format „Meet the Expert“: Gemeinsam lösen Teilnehmende und das interdisziplinäre Expertenpanel schwierige radiologische Fälle. In diesem Jahr liegt der Fokus auf der muskuloskelettalen Radiologie und der Neuroradiologie. Interaktiv sind übrigens alle Sessions dank TEDAbstimmungen. Am Samstag wird es international, wenn sich alles um den aktuellen Stand im „Cardiac Imaging“ dreht. Am selben Tag kommt der radiologische Nachwuchs auf seine Kosten. In drei „Fit für den Facharzt"-Sessions steht der Abdomen im Mittelpunkt. Darüber hinaus bietet der Fortbildungskongress ein umfangreiches Programm, mit onkologischer Bildgebung und Interventioneller Radiologie, Thoraxund Gefäßdiagnostik sowie Sportverletzungen. Zudem werden die Auswirkungen des neuen Strahlenschutzgesetzes auf die Radiologie behandelt. Natürlich ist auch wieder ein breites Angebot für alle MTRA dabei - von Diagnosen des Magen-DarmTraktes bis hin zu Untersuchungen von Hand und Ellenbogen.

Erstmalig in diesem Jahr ist das Kongresscatering im Ticketpreis inklusive. Es umfasst ein leichtes Mittagessen und kalte Getränke an allen Kongresstagen. Weitere praktische Tipps zur Orientierung vor Ort sowie Anreise mit der Bahn, dem Auto oder dem Flugzeug finden Sie unter www.radiologiekongressruhr.de > Informationen
Last but not least erwartet Sie am ersten Kongressabend ein entspanntes Get-Together im eleganten Restaurant Rosenterrassen direkt neben dem Kongresszentrum Westfalenhallen. Genießen Sie regionale und internationale Spezialitäten und kommen Sie mit Kolleginnen und Kollegen in stilvollem Ambiente ins Gespräch.

\section{RadiologieKongressRuhr 2018}

8. bis 10 . November 2018

Kongresszentrum Westfalenhallen Dortmund

www.radiologiekongressruhr.de

\section{Ihre Vorteile einer DRG-Mitgliedschaft}

Inhaltlich MITGESTALTEN Aktive Beteiligung an der inhaltlichen Arbeit und Entwicklung des Fachs in Arbeitsgemeinschaften, Foren und Programmen.

Umfassend INFORMIEREN

Immer up to date durch Publikationen, Periodika und News-Services.

\section{Persönlich VERNETZEN}

Fachlicher Austausch zu aktuellen Themen im Mitgliederbereich von drg.de, auf Veranstaltungen und Arbeitstreffen.

\section{Bedarfsbezogen NUTZEN}

Angebote und Leistungen in Anspruch nehmen wie z. B. die Zertifizierungsprogramme der DRG und ihrer Arbeitsgemeinschaften.

\section{Praktisch ORGANISIEREN}

Zusatzfunktionen und Services nutzen wie z. B. den individualisierten RöKo-Kongressplaner.

\section{Finanziell PROFITIEREN}

Weniger bezahlen bei einer Teilnahme am Deutschen Röntgenkongress und weiteren Veranstaltungen der DRG, für Mitgliedschaften in internationalen Vereinigungen, den Bezug von Zeitschriften und für Produkte unserer Kooperationspartner.

( $($ Weiterführende Informationen finden Sie hier: www.drg.de > Mitglieder 